\title{
Mineralocorticoid Receptor Overexpression Differentially Modulates Specific Phases of Spatial and Nonspatial Memory
}

\author{
Deveroux Ferguson ${ }^{1}$ and Robert Sapolsky ${ }^{1,2}$ \\ ${ }^{1}$ Department of Biological Sciences, Stanford University, Stanford, California 94305, and 2Department of Neurology and Neurological Sciences, Stanford \\ University School of Medicine, Palo Alto, California 94304
}

\begin{abstract}
Glucocorticoids (GCs) and stress modulate specific phases of information processing. The modulatory affects of GCs on hippocampal function are thought to be mediated by the mineralocorticoid receptor (MR) and the glucocorticoid receptor (GR). The GR plays a critical role in mediating the impairing effects of GCs on hippocampal function. Conversely, activation of MR facilitates hippocampal function. The high affinity of MR for GCs suggests that the receptor protein levels play a key role in regulating the beneficial effects of MR-mediated gene transcription. Using herpes simplex vectors, we transiently increased MR levels in dentate gyrus granule cells, which in turn enhanced MR signaling. We then examined its effects on spatial and nonspatial memory consolidation and retrieval using the object placement and object recognition task. Additionally, we assessed whether an increased MR signal could block the impairing effects of high GCs on memory retrieval. Rats overexpressing MR displayed an enhancement in the consolidation of nonspatial memory relative to rats expressing green fluorescent protein and suggest the potential for gene transfer techniques for enhancing cognition during stress. Moreover, rats overexpressing MR were spared from the disruptive effects of high GCs on the retrieval of nonspatial memory. Thus, this study illustrates the critical role of MR in mediating the retrieval and consolidation of nonspatial memory.
\end{abstract}

Key words: mineralocorticoid receptor; nonspatial memory; viral vector; spatial memory; corticosterone; learning and memory

\section{Introduction}

When homeostasis is threatened by stress, the body undergoes a myriad of changes. One primary physiological change is the release of glucocorticoids (GCs) by the adrenal glands. The hippocampus is a major site for such receptors, specifically the highaffinity mineralocorticoid receptor (MR), which is heavily occupied and activated at resting basal conditions, and the loweraffinity glucocorticoid receptor (GR) (de Kloet, 2000), which is primarily activated during stress, when GC levels are high.

Corticosteroid receptors for GCs occur in the hippocampus, and the interaction of GC with its ligand-dependent hormonal transcription factor (MR or GR) plays a crucial role in hippocampal synaptic plasticity and cognition (Kim and Diamond, 2002). Stress and subsequent elevated GC levels acting via the GR inhibit hippocampal long-term potentiation (LTP) and primed burst potentiation (Foy et al., 1987; Diamond et al., 1992; Pavlides et al., 1995). In contrast, MR activation enhances the induction of LTP in hippocampus (Pavlides et al., 1993; Joëls et al., 2004). In addition, MR signaling can enhance performance on spatial hippocampal-dependent cognitive tasks (de Kloet et al., 1999). However, chronic blockade of MR impairs spatial memory (Douma et al., 1998).

\footnotetext{
Received Dec. 9, 2006; revised June 18, 2007; accepted June 19, 2007.

This work was supported by National Institutes of Health Grant R01 AG020633 and National Research Service Award Predoctoral Fellowship F31 MH07346401. We thank Drs. Ted Dumas and Victoria Luine for advice on this study.

Correspondence should be addressed to Deveroux Ferguson, Gilbert Laboratory, Mail Code 5020, Stanford, CA 94305-5020. E-mail: dever089@stanford.edu.

DOI:10.1523/JNEUROSCI.1187-07.2007

Copyright $\odot 2007$ Society for Neuroscience $\quad$ 0270-6474/07/278046-07\$15.00/0
}

The use of viral vectors has become an important tool to study and manipulate rodent behavior. Decreasing GR signaling via a viral-vector-mediated strategy attenuates the impairing effects of GR activation on cognition (Nicholas et al., 2006). However, the Nicholas et al. study used a task-aversive model of hippocampaldependent spatial memory (the Morris water maze) that is intrinsically stressful, which in turn creates potential performance variables affecting motivation, arousal, and fear, all of which are known to interact with the stress pathway (de Quervain et al., 1998). Therefore, in this study, we used the spatial object placement (OP) task and the nonspatial object recognition (OR). The spontaneous preference nature of the OR and OP task contrasts with the Morris water maze because, in the former case, rats do not have to be trained using a reward contingency, thereby mitigating various performance variables.

The high affinity of MR for corticosterone (CORT) $(10 \times$ that of GR) and tonic heavy occupation suggest that receptor protein levels can play a rate-limiting role in regulating the positive effects of MR-mediated gene transcription, whereas the extent of GR signaling primarily depends on ligand concentrations (de Kloet, 2000). In this study, we assess whether using a herpes simplex virus (HSV) viral-vector-mediated strategy to overexpress MR in the dentate gyrus could block the disruptive effects of high GC levels on spatial and nonspatial hippocampal-dependent cognition. This approach offers different advantages than the use of transgenic overexpression of MR because of the compensatory developmental confounds possible in transgenic models (Oitzl et al., 2001). An alternative is the pharmacological use of MR agonists; however, these do not alter the rate-limiting step of MR levels. Indeed, rats overexpressing MR displayed an enhancement 


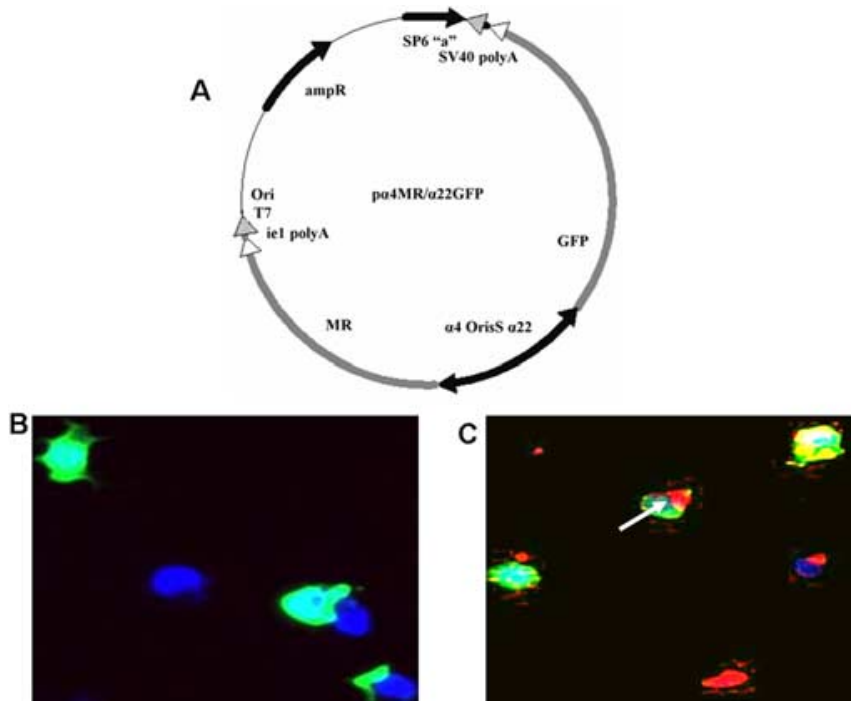

Figure 1. A, Schematic representation of the MR amplicon $p \alpha 4 M \operatorname{R} \alpha 22$ GFP. The transcriptional units MR and GFP are driven by the HSV $\alpha 4$ and $\alpha 22$ promoters, respectively, and terminated by ie1-poly(A) and simian virus 40 (SV40) poly(A) signals, respectively. The oris and " $a$ " sequences from HSV-1 provide the necessary replication signals. The control amplicon, p $\alpha 22$ GFP, is similar to $p \alpha 4 M R \alpha 22$ GFP, except the MR and ie1-poly(A) sequences are not included. $\boldsymbol{B}, \boldsymbol{C}$, COS-7 cells infected with $\mathrm{p} \alpha 22 \mathrm{GFP}$ vector expressing GFP control or $p \alpha 4 M R \alpha 22$ GFP vector expressing GFP and MR, respectively. Cells infected with p $\alpha 4 M R \alpha 22$ GFP vector overexpress the MR, and cells infected with $p \alpha 22$ GFP control vector lacked MR expression. MR immunofluorescence was visualized using a Texas Red-conjugated secondary antibody (red), nuclear staining using DAPI (blue), and infected cells by GFP expression (green). Arrows indicate nuclear localization of MR.

in the consolidation of nonspatial memory relative to green fluorescent protein (GFP) rats. Moreover, rats overexpressing MR were spared from the disruptive effects of high CORT on nonspatial memory retrieval.

\section{Materials and Methods}

Vector/plasmid construction. The MR plasmid was constructed by cloning the human MR sequence from the PHMR3750 (courtesy of Dr. Ron Evans, The Salk Institute for Biological Studies, La Jolla, CA) plasmid (GenBank accession number m80582) into a p $\alpha 22 \mathrm{eGFP}-4 \alpha$ plasmid (Fig. 1A) described previously (Kaufer et al., 2004). The human MR sequence was isolated by PCR amplification with the addition of HindIII sticky ends to facilitate ligation into the backbone of p $\alpha 22 \mathrm{eGFP}$. Once constructed, the $\mathrm{p} \alpha 22 \mathrm{eGFP}-\mathrm{p} 4 \alpha \mathrm{MR}$, a bipromoter plasmid, expresses MR and GFP protein with a $98 \%$ covariance (Fink et al., 1997). The control construct, $\mathrm{p} \alpha 22 \mathrm{eGFP}$, consisted of a plasmid expressing only GFP. Constructs were packaged and amplified into HSV particles.

Generation of viral vectors. Viral vectors were produced as described previously (Ho, 1994). Transient transfection of plasmids into E5 cells (African green monkey Vero cells stably transfected with the HSV $\alpha 4$ gene required for propagation) was followed $24 \mathrm{~h}$ later by superinfection with the helper virus d120, a defective virus required for the replication and packaging of the amplicon. Once complete cytopathic effect was observed, amplicons were harvested by freeze-thaw lysis followed by sucrose cushion centrifugation at 20,000 $\times g$ overnight. Pelleted viruses were resuspended in $\mathrm{ddH} 2 \mathrm{O}$ and frozen at $-80^{\circ} \mathrm{C}$. Superinfection gives rise to vector titers of $2-3 \times 10^{6}$ infectious particles/ml (p $\alpha 22$ eGFP and $\mathrm{p} \alpha 22 \mathrm{eGFP}-\mathrm{p} 4 \alpha \mathrm{MR})$ and $\mathrm{d} 120$ helper virus titers of $0.4-1.0 \times 10^{7}$ plaque forming units $(\mathrm{PFU}) / \mathrm{ml}$.

Adrenalectomy. One week after arrival to our animal facility, male Long-Evans rats weighting 200-400 g (Charles River Laboratories, Wilmington, MA) were adrenalectomized (ADX). Animals were allowed $5 \mathrm{~d}$ to recover before any additional surgeries were performed.

Sterotaxic surgery. Animals were anesthetized with a ketamine/xylazine/acepromazine mixture for stereotaxic intracranial delivery of virus.
A $10 \mu \mathrm{l}$ Hamilton syringe connected to a 28 gauge injection needle was used to infuse virus. Enhanced GFP (eGFP) or MR-expressing viral particles were delivered to the dentate gyrus (DG) of the hippocampus $(-3.5$ $\mathrm{mm}$ anteroposterior and $\pm 2.5 \mathrm{~mm}$ mediolateral from bregma, $-2.5 \mathrm{~mm}$ dorsoventral from dura at $0.150 \mu \mathrm{l} / \mathrm{min}$ ) $3 \mathrm{~d}$ before behavioral testing. All injections for behavioral studies were bilateral.

Corticosterone injections. Animals received an acute injection of a "high CORT (10 mg/kg)" solution, which generates sustained stress CORT levels $(\sim 28 \mu \mathrm{g} / \mathrm{dl})$ for $\sim 20 \mathrm{~h}$ (Stein-Behrens et al., 1994), or "low CORT," which consisted of a vehicle injection of peanut oil administered either immediately preceding the sample phase or $30 \mathrm{~min}$ before the test phase. To assess the effects of CORT on the consolidation of nonspatial memory, all animals were injected once with low or high CORT immediately after the sample phase. The effects of CORT on the retrieval of nonspatial memory was assessed in a different group of animals by injecting them once with low (vehicle) or high CORT 30 min before testing at the $24 \mathrm{~h}$ delay, producing $28 \mu \mathrm{g} / \mathrm{dl}$ CORT at the time of testing (SteinBehrens et al., 1994). Experimentation reported was conducted in accordance with the guidelines specified by the National Institutes of Health (NIH Guide for the Care and Use of Laboratory Animals) and the Stanford Department of Veterinary Services and Care.

Object recognition testing. Testing was performed in an open-field arena $(60 \times 60 \times 45 \mathrm{~cm})$ constructed of black Plexiglas to reduce distal cues. The stimulus objects varied in shape and color and were made of glass, plastic, or ceramic. The sizes of the objects were no smaller than the size of the rat and no larger than $\sim 2.5$ times the size of the rat. Most objects were heavy enough that they could not be moved by the rats. At the beginning of a trial, each rat was placed in the empty arena and allowed to explore for $1 \mathrm{~min}$. Rats were then removed and placed in a transport box for $30 \mathrm{~s}$, and two identical objects were placed in the arena. Trials consisted of a sample trial and a recognition trial. The two trials were separated by an intertrial interval of varying lengths. In the sample phase, two identical objects were placed at one end of the open field, and amount of time spent exploring the two objects was recorded. To assess the effects of CORT on the consolidation of nonspatial memory, all animals were injected once with low or high CORT immediately after the sample phase. For the recognition trial, a new object replaced one of the previous objects, and time spent exploring the old (familiar object) and the new (novel) object was recorded. The effects of CORT on the retrieval of nonspatial memory was assessed in a different group of animals by injecting animals once with low (vehicle) or high CORT $30 \mathrm{~min}$ before testing at the $24 \mathrm{~h}$ delay (producing circulating concentrations of 25-30 $\mu \mathrm{g} / \mathrm{dl}$ CORT at the time of testing). The objects used for trials were various bottles, cans, and containers (such as soda cans and candlesticks). Delays of 1 and $24 \mathrm{~h}$ were used between sample and test phase. Separate groups of rats were tested at the 1 or $24 \mathrm{~h}$ delay. Location and the novel object were counterbalanced (left or right) across the trials. Object exploration was scored only when the rat's nose was within $2 \mathrm{~cm}$ of the object and the vibrissae were moving. A stop watch was used to collect and analyze the behavioral data during both the sample and test phases. Sessions were videotaped and later analyzed.

Object-placement task. Object placement is a hippocampal-dependent spatial memory task (Li et al., 2004). The current method for object placement testing in rats was adapted from use in mice (Luine et al., 2003). Rats were placed on the field with two identical objects for 5 min. Exploration of the objects was timed by a stop watch when rats sniffed, whisked, or looked at the objects from no more than $2 \mathrm{~cm}$ away. After the sample phase, the rats were removed from the field for $15 \mathrm{~min}$. After the delay, one object was moved to a new location. The time spent exploring the objects in new (novel) and old (familiar) locations were recorded. Sessions were videotaped and later analyzed. All locations for the objects were counterbalanced among groups.

Histology. Immediately after behavioral assay, animals were decapitated and perfused transcardially with heparinized saline and a $4 \%$ paraformaldehyde (PFA) solution. Brains were removed and stored overnight in a $4 \% \mathrm{PFA} / 30 \%$ sucrose solution and sectioned at $30 \mu \mathrm{m}$ thickness on a cryostat. Granule cells were visualized using a fluorescence microscope at $490 \mathrm{~nm}$ excitation and $10-20 \times$ magnification.

Tissue culture procedure. Tissue culture was performed as published 
previously (Brooke et al., 1997). Briefly, hippocampi were removed from 18-d-old fetal rats. Cells were dissociated with papain, filtered through an $80 \mu \mathrm{m}$ cell strainer, and resuspended in modified MEM (University of California, San Francisco, Tissue Culture Facility) containing $30 \mathrm{~mm}$ glucose and $10 \%$ horse serum (HyClone, Logan, UT). Cells were plated at a density of 187,500 cells $/ \mathrm{cm}^{2}$ on 96 -well plates or on T75 flasks at a density $5 \times 105$ cells $/ \mathrm{cm}^{2}$. The cells were used between days 10 and 12 in culture. Mixed cultures consist of 5\% microglia, $20-30 \%$ of neurons, and $65-75 \%$ other cell types of glia.

Fluorescent immunocytochemistry. Primary hippocampal cultures and COS-7 cells were infected with the viral vectors, as described previously (Kaufer et al., 2004). Cells were fixed with $4 \%$ paraformaldehyde and incubated overnight at $4^{\circ} \mathrm{C}$ with a $1: 200$ dilution of a polyclonal anti-MR antibody (Affinity BioReagents, Golden, $\mathrm{CO}$ ) and then for $2 \mathrm{~h}$ at room temperature in a 1:250 dilution of Texas Redconjugated secondary antibody (goat antirabbit Ig; Vector Laboratories, Burlington, CA) and 4,6-diamidino-2-phenylindole (DAPI) for nuclear staining. Fluorescent microscope images were taken in the wavelengths corresponding to GFP expression, MR expression, and the nuclear stain.

Mineralocorticoid receptor ELISA. Functional assays such as an electrophoretic mobility shift assay (EMSA) are often hampered by limited quality and quantity of the material. The MR ELISA is significantly more sensitive than EMSA and allows determination of DNA-binding activity of whole-cell extract from tissue samples. Protein extracts from the dentate gyrus of rats expressing GFP control or overexpressing MR vector were prepared using the Nuclear Extract kit (Active Motif, Carlsbad, CA) according to the instructions of the manufacturer. MR activity was determined using TransAM kit from Active Motif according to the instructions of the manufacturer. All reactions were performed in duplicate. As a positive control, $20 \mu \mathrm{l}$ of complete lysis buffer containing $1 \mu \mathrm{l}$ of positive control cell extract per well was used. For negative control, $20 \mu \mathrm{l}$ of complete lysis buffer per well was used. Briefly, whole cell extracts (5-10 $\mu \mathrm{g})$ from GFP control and rats overexpressing MR were added in duplicate to 96-well plates precoated with the oligonucleotide containing GRE consensus sequence ( $5^{\prime}$ GGTACANNNTGTTCT-3'). The plate was sealed with adhesive film and incubated for $1 \mathrm{~h}$ at room temperature with mild agitation (100 rpm on a rocking platform), after which the wells were washed three times with $200 \mu \mathrm{l}$ of wash buffer and $100 \mu$ l of diluted primary antibody (1:250 dilution in antibody-binding buffer) was added to each well and incubated at room temperature for $1 \mathrm{~h}$ without agitation. The wells were washed again three times with wash buffer, and $100 \mu \mathrm{l}$ of diluted horseradish peroxidase-conjugated antibody (1:1000 dilution in antibody-binding buffer) was added to each well and incubated for $1 \mathrm{~h}$ at room temperature. The wells were washed again four times with wash buffer followed by the addition of $100 \mu \mathrm{l}$ of developing solution. The wells were incubated for $10 \mathrm{~min}$ at room temperature shielded from direct light. This was followed by the addition of $100 \mu$ l of stop solution to each well, and the absorbance was read within $5 \mathrm{~min}$ at $450 \mathrm{~nm}$. MR levels were quantified relative to GFP controls at 3 and $4 \mathrm{~d}$ after vector infusion.

Statistics. Fisher's least significant difference (LSD) post hoc was performed after significant results from ANOVAs. For object recognition and placement trials, one-way ANOVAs tested for differences among groups in exploration time during sample phase. For the test trial, twoway ANOVAs was performed to assess group differences (control or transgene) $\times$ object (old or new). Paired $t$ tests on each group tested was used to determine whether time spent with the (novel) object or location was greater than time spent with the old object or location (familiar). If
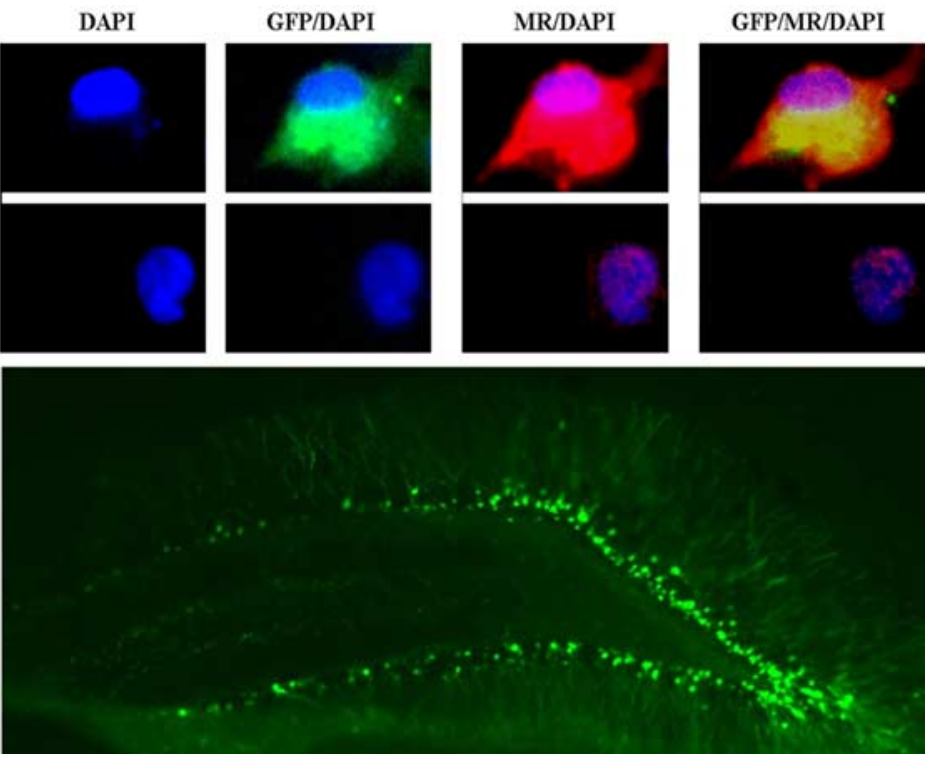

Figure 2. A, Mineralocorticoid expression in primary hippocampal cultures. Cells infected with p $\alpha 4 \mathrm{MR} \alpha 22 \mathrm{GFP}$ vector overexpressing MR protein to a greater degree when compared with uninfected primary hippocampal cultures. MR immunofluorescence was visualized using a Texas Red-conjugated secondary antibody (red), nuclear staining using DAPI (blue), and infected cells by GFP expression (green). $\boldsymbol{B}$, Digital images showing robust in vivo expression of GFP-tagged MR transgene $72 \mathrm{~h}$ after infusion. Expression of both MR and GFP vectors was evident throughout the dentate gyrus subfield of the hippocampus.

subjects spent significantly more time exploring the new object or location, they were considered to have discriminated and remembered. Twoway ANOVAs were used to detect group differences in the ELISA study.

\section{Results}

Overexpressed MR demonstrates nuclear translocation in hippocampal and COS-7 cells

To validate functional overexpression of MR, COS-7 cells were infected with $\mathrm{p} \alpha 22 \mathrm{GFP}$ vector expressing GFP control or p $\alpha 4 \mathrm{MR} \alpha 22 \mathrm{GFP}$ vector expressing MR and reporter GFP. Cells infected with $\mathrm{p} \alpha 22 \mathrm{GFP}$ control vector lacked MR expression (Fig. $1 B$ ), whereas cells infected with $\mathrm{p} \alpha 4 \mathrm{MR} \alpha 22 \mathrm{GFP}$ vector demonstrated significant overexpression of the mineralocorticoid receptor (Fig. 1C). Arrows indicate nuclear localization of $\mathrm{MR}$, demonstrating that overexpressed MR retains its capacity for nuclear translocation.

Infection of primary hippocampal culture cells with p $\alpha 4 \mathrm{MR} \alpha 22 \mathrm{GFP}$ vector resulted in greater MR protein levels when compared with uninfected primary hippocampal cultures. MR immunofluorescence was visualized using a Texas Redconjugated secondary antibody (red), nuclear staining using DAPI (blue), and infected cells by GFP expression (green) (Fig. $2 A$ ). Digital images show robust in vivo expression of GFP-tagged MR transgene $72 \mathrm{~h}$ after infusion. Expression of $\mathrm{p} \alpha 4 \mathrm{MR} \alpha 22 \mathrm{GFP}$ vector was evident throughout dentate gyrus subfield of the hippocampus (Fig. $2 B$ ).

MR protein levels are elevated 3 and $4 \mathrm{~d}$ after vector infusion The two phases of the object recognition test were performed 3 and $4 \mathrm{~d}$ after vector infusion. Using an MR ELISA assay, we quantified MR levels in GFP control rats and rats overexpressing $M R$ vector $3 \mathrm{~d}$ and $4 \mathrm{~d}$ after vector infusion. A two-way ANOVA revealed a significant increase in MR levels at both time points in rats overexpressing MR vector $(p<0.05)$ relative to GFP control animals (Fig. 3). 


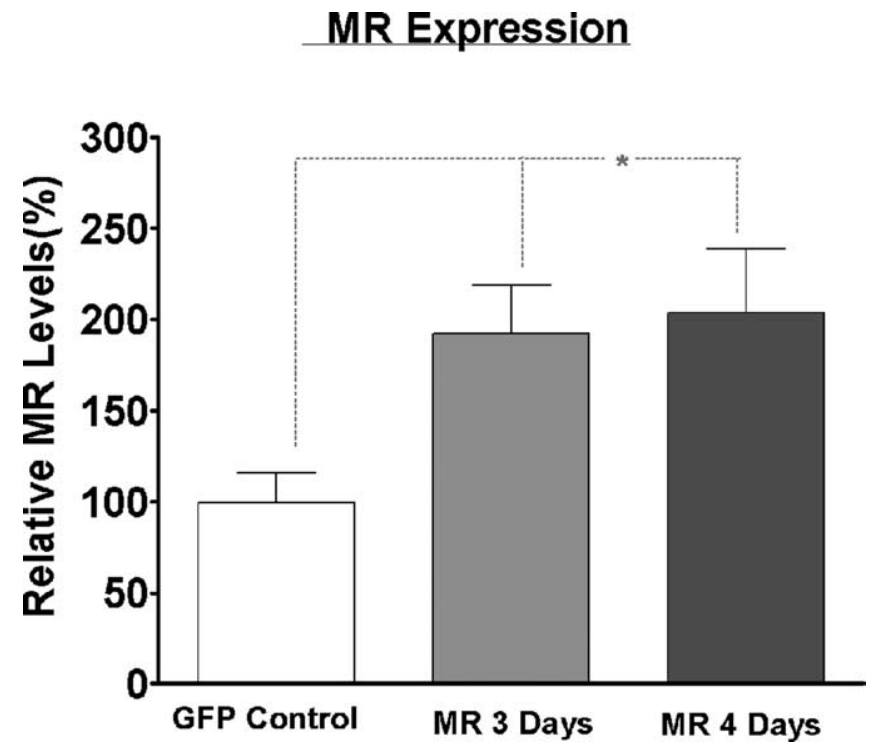

Figure 3. ELISA-based MR overexpression assay. Analysis of tissue sample extracts from rats expressing GFP control or MR vector 3 or $4 \mathrm{~d}$ after vector infusion. There was a significant increase in MR levels in rats infused with MR vector at both 3 and $4 \mathrm{~d}$ after vector infusion relative to rats expressing GFP control vector. Data are represented as mean \pm SEM; GFP $3 \mathrm{~d}$, $n=11 ; \mathrm{MR} 3 \mathrm{~d}, n=11 ; \mathrm{GFP} 4 \mathrm{~d}, n=11 ; \mathrm{MR} 4 \mathrm{~d}, n=7$. Two-way ANOVA with posthoc analysis. ${ }^{*} p<0.05$ compared with GFP control.

\section{HR OBJECT RECOGNITION}
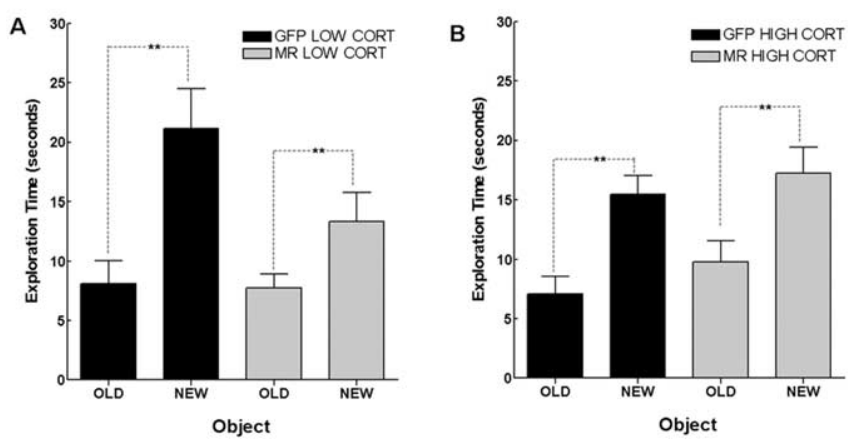

Figure 4. Effects of MR overexpression on object recognition memory. One hour delay exploration analysis during the test phase of the object recognition task for low CORT (vehicle peanut oil, subcutaneously) $(\boldsymbol{A})$ and high CORT (10 $\mathrm{mg} / \mathrm{kg}$, s.c.) ( $\boldsymbol{B})$ expressing GFP or MR vector ( $n=10-11$ per group). Data represent exploration of old or novel object at a $1 \mathrm{~h}$ retention trial expressed as mean \pm SEM. $A$, Low CORT GFP and low CORT MR rats significantly discriminate at the $1 \mathrm{~h}$ retention trial. $\boldsymbol{B}$, Treatment with high CORT ( $10 \mathrm{mg} / \mathrm{kg}$, S.c.) does not effect the object recognition memory in rats expressing GFP or MR vector. Rats expressing GFP or MR treated with high CORT can discriminate at the $1 \mathrm{~h}$ delay. Paired $t$ test, ${ }^{* *} p<0.01$.

\section{MR overexpression enhances object recognition memory consolidation}

During sample trials, no significant differences between groups were found in the time spent exploring identical objects $(p>0.1$; data not shown). To assess the effects of CORT on the consolidation of nonspatial memory, animals were injected with low or high CORT immediately after the sample phase. All animals were able to discriminate between old and novel objects after a $1 \mathrm{~h}$ delay (Fig. 4). Figure 4, $A$ and $B$, shows that low CORT or high CORT treatment did not effect object recognition memory with a $1 \mathrm{~h}$ (short) delay between the sample-to-test phase; both low CORT $(p<0.01)$ and high CORT $(p<0.05)$ groups spent significantly greater time exploring the novel object than the previously explored (familiar) object.

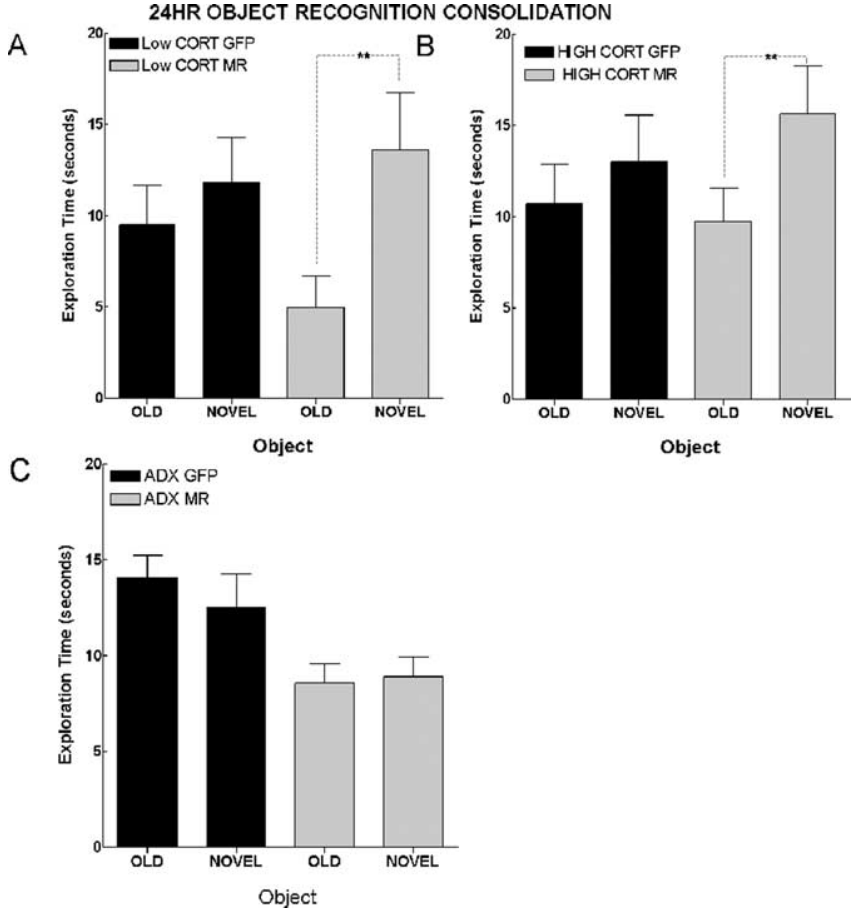

Figure 5. Twenty-four hour object recognition memory consolidation during the test phase of the object recognition task for low CORT (vehicle peanut oil, subcutaneously) $(\boldsymbol{A})$, high CORT $(10 \mathrm{mg} / \mathrm{kg}, \mathrm{s.c.}$.) (B), and adrenalectomized animals ( $\boldsymbol{C}$ ) expressing GFP or MR vector $(n=9-11$ per group). Data represent time spent exploring the old or novel object (mean \pm SEM). $\boldsymbol{A}, \boldsymbol{B}$, GFP animals with low or high circulating levels of CORT were incapable of discriminating at the $24 \mathrm{~h}$ delay. In contrast, animals overexpressing MR outperformed their GFP controls spending significantly more time exploring the novel object than the old one whether treated with high or low CORT (low CORT MR old vs low CORT MR novel, $p=0.005$; high CORT MR old vs high CORT MR novel, $p=0.003$ ). C, Adrenalectomized animals expressing GFP or MR vector spent an equivalent amount of time exploring a novel object and a previously encountered familiar object. Paired $t$ test, ${ }^{* *} p<0.01$.

In contrast to the situation at the time of a $1 \mathrm{~h}$ delay, group differences emerged when testing was conducted with a $24 \mathrm{~h}$ delay. Rats expressing GFP control vector and injected with vehicle, thus having basal circulating CORT levels (low CORT), were impaired in their ability to discriminate between old and novel objects, spending an equivalent amount of time exploring both the previously encountered object and the novel object after a $24 \mathrm{~h}$ delay (Fig. 5A). In contrast, rats overexpressing the MR vector and injected with vehicle (low CORT) were able to discriminate at the $24 \mathrm{~h}$ delay, spending significantly more time exploring the novel object versus the old object (Fig. $5 A)(p=$ 0.005). Fisher's LSD post hoc after a two-way ANOVA revealed that rats expressing MR vector and treated with low CORT spent significantly $(F=6.896 ; p=0.011)$ more time exploring novel object relative to rats expressing GFP vector and treated with low CORT. In addition, rats overexpressing MR and injected with high CORT showed an enhancement in performance displaying a 1.5 -fold increase in preference for the novel object when compared with rats expressing GFP and injected with high CORT (Fig. $5 B)(p=0.003)$. To verify that the effects of MR overexpression were directly attributable to CORT binding to MR, experiments using ADX animals were performed. Comparison of performance in ADX-GFP and ADX-MR revealed no difference in performance (Fig. 5C); hence, there has to be a concomitant activation of the ligand-dependent hormonal transcription factor (MR) by its ligand (GC). 
A

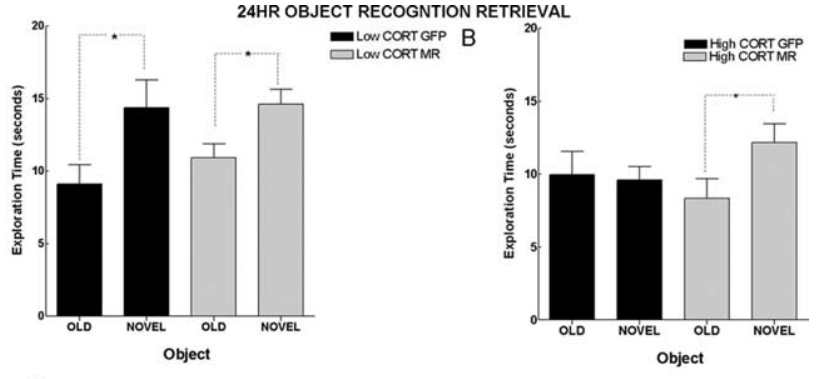

C

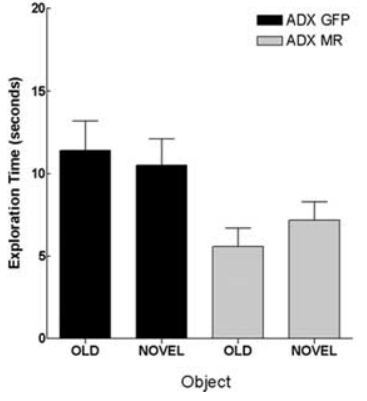

Figure 6. Effects of MR overexpression on object recognition memory retrieval. Data represent exploration of old or novel object at a $24 \mathrm{~h}$ retention trial expressed as mean $\pm S E M$. $A$, Low CORT GFP and low CORT MR rats significantly discriminate at the $24 \mathrm{~h}$ retention trial (Iow CORT GFP old vs low CORT GFP novel, $p=0.033$; low CORT MR old vs low CORT MR novel, $p=0.011$; $n=9-23$ per group). $\boldsymbol{B}$, Treatment with high CORT (10 mg/kg, s.c.) impairs the retrieval of object recognition memory in rats expressing GFP. Rats overexpressing MR are spared from the impairing effects of high CORT on the retrieval of object recognition memory (high CORT GFP old vs high CORT GFP novel, $p=0.827$; high CORT MR old vs high CORT MR novel, $p=0.015 ; n=$ 11-16 per group). Paired $t$ test, ${ }^{*} p<0.05$. C, ADX animals spent an equivalent amount of time exploring both old and novel objects. No significant group differences were observed. Paired $t$ test.

\section{MR overexpression attenuates the impairing effects of CORT} on memory retrieval

Next we determined the effects of MR overexpression and CORT administration on memory retrieval. During sample trials, no significant differences between groups (low and high CORT or overexpressing GFP or MR) were found in the time spent exploring identical objects (data not shown). All animals were injected with low (vehicle) or high CORT $30 \mathrm{~min}$ before testing at the $24 \mathrm{~h}$ delay to assess the effects of CORT on memory retrieval. MR- or GFP-expressing rats injected with low CORT 30 min before testing on the $24 \mathrm{~h}$ retention trial were able to discriminate between a previously encountered (old) object and a novel object (Fig. $6 A)$. Treatment with high CORT $(10 \mathrm{mg} / \mathrm{kg}$, s.c.) impaired the retrieval of object recognition memory in rats expressing GFP (Fig. $6 B$ ). In contrast, rats overexpressing MR were spared from the impairing effects of high CORT (Fig. $6 B)$ ( $p=0.015)$. Comparison of performance in ADX-GFP and ADX-MR revealed no difference in performance (Fig. 6C).

MR overexpression does not attenuate the impairing effects of CORT on short-term object placement memory

We next assessed whether the beneficial effects of MR overexpression extend to other hippocampal-dependent cognitive task. Animals expressing GFP or MR were injected with low (vehicle) or high CORT 15 min before testing on the retention trial. Spatial memory was assessed using the object placement task (Luine et al., 2003). During sample trials, no significant differences between groups (low and high CORT or GFP and MR) were found in the time spent exploring identical objects (data not shown). Low CORT GFP and low CORT MR rats significantly discrimi-
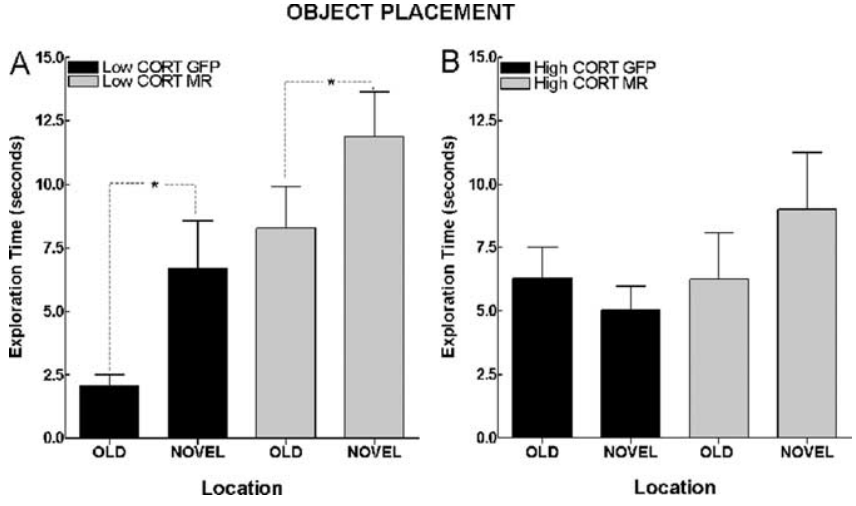

Figure 7. MR overexpression does not attenuate the impairing effects of high CORT on the spatial object placement task. $A$, Low CORT GFP and low CORT MR rats significantly discriminate at the 15 min retention trial (low CORT GFP old vs low CORT GFP novel, $p=0.019$; low CORT MR old vs low CORT MR novel, $p=0.016 ; n=9$ per group). Data represent exploration time of old or novel object location at a 15 min retention trial expressed as mean \pm SEM. Paired $t$ test, ${ }^{*} p<$ 0.05. B , Treatment with high CORT ( $10 \mathrm{mg} / \mathrm{kg}$, s.c.) impairs object placement memory in rats expressing GFP and MR. Rats overexpressing MR are not protected from the disruptive effects of high CORT treatment on spatial object placement memory (high CORT GFP old vs high CORT GFP novel, $p=0.230$; high (ORT MR old vs high (ORT MR novel, $p=0.416 ; n=5-11$ per group). Paired $t$ test.

nated at the 15 min delay (Fig. 7A). In contrast, treatment with high CORT (10 mg/kg, s.c.) impaired the retrieval of object placement memory in rats, whether they expressed GFP or MR (Fig. 7B).

\section{Discussion}

In this present study, we assess whether using an HSV viralvector-mediated strategy to overexpress $M R$ in the dentate gyrus could block the disruptive effects of stress level GCs on nonspatial and spatial hippocampal-dependent cognition using the OR and the OP task. Rats overexpressing MR displayed an enhancement in the consolidation of nonspatial memory relative to rats expressing GFP. More importantly, rats overexpressing MR were spared from the disruptive effects of high GCs on the retrieval of nonspatial memory. In contrast, MR overexpression was less effective on the more difficult spatial memory task. A recent paper examined the role of MR in corticosteroid-mediated regulation of the hypothalamic-pituitary-adrenal (HPA) axis and of anxiety-like behaviors, using transgenic mice that overexpressed MR in the forebrain. The study revealed that such overexpression reduced anxiety-like behavior in both male and female mice. These findings suggest that MR acts to counterbalance GR in the modulation of anxiety-related behaviors (Rozeboom et al., 2007). Similar to the findings regarding anxiety in that study, we demonstrated that MR overexpression in the dentate gyrus counterbalances the impairing effects of GR modulation on nonspatial memory retrieval.

\section{MR overexpression enhances nonspatial object recognition memory consolidation}

The role of the hippocampus in nonspatial memory is controversial (Mumby, 2001); however, abundant new data suggest that the hippocampus plays a central role in object recognition performance (Clark et al., 2000; Baker and Kim, 2002; Broadbent et al., 2004). Our study suggests that overexpression of MR enhances OR memory consolidation at a $24 \mathrm{~h}$ delay. An ELISA for MR demonstrated robust MR overexpression 3 and $4 \mathrm{~d}$ after vector infusion (Fig. 3 ); the 3 and $4 \mathrm{~d}$ time points correspond to the sample phase and $24 \mathrm{~h}$ test phase behavioral time points. 
Consolidation is a slow protein-dependent process (Wang et al., 2006); therefore, studies assessing the relationship of GCs and MR overexpression on this phase of information processing require several hours of delay between treatment and testing. Rats overexpressing MR or GFP were injected with either high or low CORT immediately after the sample phase and then tested for object recognition memory 1 or $24 \mathrm{~h}$ later. Our analysis revealed that, during the OR task, low CORT rats expressing GFP performed adequately with a $1 \mathrm{~h}$ retention delay (Fig. $4 A$ ) but were impaired at the longer $24 \mathrm{~h}$ delay (Fig. 5A). This finding is in agreement with the demonstration that vehicle-treated rats were able to discriminate at the easier $1 \mathrm{~h}$ delay but were impaired at the more difficult $24 \mathrm{~h}$ delay (Okuda et al., 2004). In contrast, low CORT rats overexpressing MR displayed a significant preference for the novel object at the longer $24 \mathrm{~h}$ delay (Fig. 5A), suggesting that an increased MR signaling positively modulates performance. In addition, high CORT rats overexpressing MR showed enhanced performance relative to high CORT rats expressing GFP (Fig. 5B). To our knowledge, this is the first study demonstrating that overexpression of MR in the dentate gyrus specifically enhances nonspatial memory consolidation; these results are supportive of other studies demonstrating that MR activation positively modulates cognitive function. For example, Conrad et al. (1997) showed that spatial recognition memory on the Y-maze can be selectively improved in ADX rats by activating MR using aldosterone. Conversely, central administration of spironolactone impaired performance in the water maze, decreasing time spent in the goal quadrant (Oitzl and de Kloet, 1992; Yau et al., 1999).

Although mild exposure to GCs or stress can facilitate hippocampal-dependent function, the impairing effects of GCs occur when GCs reach extremely high or low levels, such as those achieved by high stress or adrenalectomy (Conrad, 2006). This inverse- $U$ function is a reflection of the diversity of receptors for GCs in the hippocampus (de Kloet et al., 1993). The salutary effects of mild GC elevations are mediated by the high-affinity $\mathrm{MR}$, whereas the disruptive effects are mediated by the lowaffinity GR that is only heavily occupied in the face of high stress levels of GCs (de Kloet et al., 1993). We observed a slight trend toward an inverted-U function. In addition, we observed that low CORT rats overexpressing MR outperformed ADX rats overexpressing MR (Fig. 5). This suggests that MR overexpression is beneficial insofar as the receptor acts in its traditional manner, i.e., binding CORT. When GC levels are in the basal range, MR is heavily, but not entirely, occupied. It is only when GC levels are increased (into the low stress range) that MR levels becomes rate limiting; thus, increasing MR expression augments MR signaling when GC levels are high. This effect is consistent with a study showing that aldosterone improved the performance of ADX rats to the control level (Conrad et al., 1997). The mechanism underlying the enhanced performance may stem from the MRmediated increase in hippocampal neuronal excitability (Pavlides et al., 1994). MR agonists decrease afterhyperpolarization, thus increasing neuronal excitability (Joëls et al., 1992; Pavlides et al., 1993) and facilitating the induction of LTP and primed burst potentiation (a low-threshold form of LTP).

MR overexpression attenuates the impairing effects of CORT on nonspatial object recognition memory retrieval

Previous studies have reported that exposure of rats to stress or glucocorticoid injection impairs memory retrieval on radial-arm and water-maze spatial tasks (Roozendaal, 2003). In this second study, we assessed the therapeutic potential of MR overexpres- sion to block the impairing effects of high CORT on memory retrieval. Low CORT animals were able to discriminate when injected $30 \mathrm{~min}$ before testing (Fig. 6A) but impaired when injected immediately after the sample phase (Fig. $5 A$ ). This suggests that the injection by itself increased arousal, which facilitated memory retrieval, an idea commensurate with previous work (Conrad et al., 1997). Treatment with high CORT impaired the retrieval of object recognition memory in rats expressing GFP (Fig. 6B). These results are consistent with data indicating that glucocorticoids or stress administered to rats or humans before retention testing impair retrieval of long-term memory (de Quervain et al., 1998, 2000). Because low CORT and high CORT groups explored the identical objects to an equivalent extent during the sample phase, it is unlikely that a difference in acquisition between the groups underlies the selective impairment of high CORT on memory retrieval. In contrast, MR overexpression proved to be protective, and rats overexpressing MR were spared from the impairing effects of high CORT on the retrieval of object recognition memory (Fig. $6 B$ ). How does MR mediate these effects? It can be speculated that high MR levels in the hippocampus increase the inhibitory feedback to the HPA, axis thereby decrease the stress response (Rozeboom et al., 2007).

\section{MR overexpression does not attenuate the impairing effects of CORT on spatial object placement memory}

The observation that MR overexpression is protective against high CORT impairments in nonspatial memory retrieval led us to assess whether the protective effects of MR overexpression extended to spatial memory retrieval. The finding that rats were impaired when injected with high CORT and overexpressing GFP are in accord with those of previous studies reporting that glucocorticoid injection impairs short-term memory (Vazquez et al., 1998; Roozendaal, 2003). An explanation for the lack of a protective effect of MR overexpression may stem from the fact that spatial memory is more susceptible to hippocampal dysfunction than nonspatial memory; lesions of at least $80 \%$ of the hippocampus are necessary to impair object recognition, whereas smaller lesions of the hippocampus (30\%) impair spatial memory tasks (Broadbent et al., 2004). The spatial object placement task is more difficult than the nonspatial object recognition task, making it more susceptible to GR-mediated hippocampal dysfunction and less amenable to MR overexpression overriding the GR effects.

\section{Gene therapy to modulate cognitive function}

HSV is neurotrophic by nature preferentially infecting neurons versus glial. The fact that our viral vectors infected only a small percentage of granule cells in the dentate gyrus raises the possibility of observing a negligible downstream behavioral endpoint (cognitive function). Contrary to this, MR overexpression enhanced the consolidation of nonspatial memory and attenuating the disruptive effects of high CORT on the retrieval of nonspatial memory, showing that altering a subpopulation of neurons can be sufficient to alter an entire neuronal network. Similarly, HSV infection of just a portion of DG is sufficient to alter performance on a hippocampal-dependent spatial memory task (Dumas and Sapolsky, 2001; Dumas et al., 2004). In conclusion, using gene therapy, we disassociated the effects of an enhancement in MR signaling on spatial and nonspatial memory consolidation and retrieval, demonstrating that increasing MR signaling improves nonspatial memory consolidation and attenuates the disruptive effects of high GCs on memory retrieval. Generally, memory and learning impairments are thought to involve disturbed GR- 
mediated effects. However, MRs rather than GRs are markedly reduced in number when corticosteroid levels are elevated (Vazquez et al., 1998). We hypothesize that overexpressing MR allows us to address this imbalance, thereby attenuating the detrimental effects of excessive GR activation on cognitive function. These findings provide the groundwork for possible neuronal substrates to target once the field of cognitive gene therapy has matured.

\section{References}

Baker KB, Kim JJ (2002) Effects of stress and hippocampal NMDA receptor antagonism on recognition memory in rats. Learn Mem 9:58-65.

Broadbent NJ, Squire LR, Clark RE (2004) Spatial memory, recognition memory, and the hippocampus. Proc Natl Acad Sci USA 101:14515-14520.

Brooke S, Chan R, Howard S, Sapolsky R (1997) Endocrine modulation of the neurotoxicity of gp120: implications for AIDS-related dementia complex. Proc Natl Acad Sci USA 17:9457-9462.

Clark RE, Zola SM, Squire LR (2000) Impaired recognition memory in rats after damage to the hippocampus. J Neurosci 20:8853-8860.

Conrad CD (2006) The relationship between acute glucocorticoid levels and hippocampal function depends upon task aversiveness and memory processing stage. Nonlinearity Biol Toxicol Med 3:57-78.

Conrad CD, Lupien SJ, Thanasoulis LC, McEwen BS (1997) The effects of Type I and Type II corticosteroid receptor agonists on exploratory behavior and spatial memory in the Y-maze. Brain Res 759:76-83.

de Kloet ER (2000) Stress in the brain. Eur J Pharmacol 405:187-198.

de Kloet ER, Oitzl MS, Joëls M (1993) Functional implications of brain corticosteroid receptor diversity. Cell Mol Neurobiol 13:433-455.

de Kloet ER, Oitzl MS, Joëls M (1999) Stress and cognition: are corticosteroids good or bad guys? Trends Neurosci 22:422-426.

de Quervain DJ, Roozendaal B, McGaugh JL (1998) Stress and glucocorticoids impair retrieval of long-term spatial memory. Nature 394:787-790.

de Quervain DJ, Roozendaal B, Nitsch RM, McGaugh JL, Hock C (2000) Acute cortisone administration impairs retrieval of long-term declarative memory in humans. Nat Neurosci 3:313-314.

Diamond DM, Bennett MC, Fleshner M, Rose GM (1992) Inverted-U relationship between the level of peripheral corticosterone and the magnitude of hippocampal primed burst potentiation. Hippocampus 2:421-430.

Douma BRK, Korte SM, Buwalda B, la Fleur SE, Bohus B, Luiten PGM (1998) Repeated blockade of mineralocorticoid receptors, but not of glucocorticoid receptors impairs food rewarded spatial learning. Psychoneuroendocrinology 23:33-44.

Dumas TC, Sapolsky RM (2001) Gene therapy against neurological insults: sparing neurons versus sparing function. Trends Neurosci 24:695-700.

Dumas TC, Powers EC, Tarapore PE, Sapolsky RM (2004) Overexpression of calbindin $\mathrm{D}(28 \mathrm{k})$ in dentate gyrus granule cells alters mossy fiber presynaptic function and impairs hippocampal-dependent memory. Hippocampus 14:701-709.

Fink SL, Hang LK, Ho DY, Sapolsky RM (1997) Defective herpes simplex virus vectors expressing the rat brain stress-inducible heat shock protein 72 protect cultured neurons from severe heat shock. J Neurochem 68:961-969.

Foy MR, Stanton ME, Levine S, Thompson RF (1987) Behavioral stress impairs long-term potentiation in rodent hippocampus. Behav Neural Biol 48:138-149.

Ho DY (1994) Amplicon-based herpes simplex virus vectors. Methods Cell Biol 43:191-210.
Joëls M, de Kloet ER (1992) Control of neuronal excitability by corticosteroid hormones Trends Neurosci 15:25-30.

Joëls M, Karst H, Alfarez D, Heine VM, Qin Y, van Riel E, Verkuyl M, Lucassen PJ, Krugers HJ (2004) Effects of chronic stress on structure and cell function in rat hippocampus and hypothalamus. Stress 7:221-231.

Kaufer D, Ogle WO, Pincus ZS, Clark KL, Nicholas AC, Dinkel KM, Dumas TC, Ferguson D, Lee AL, Winters MA, Sapolsky RM (2004) Restructuring the neuronal stress response with anti-glucocorticoid gene delivery. Nat Neurosci 7:947-953.

Kim JJ, Diamond DM (2002) The stressed hippocampus, synaptic plasticity and lost memories. Nat Rev Neurosci 3:453-462.

Li C, Brake WG, Romeo RD, Dunlop JC, Gordon M, Buzescu R, Magarinos AM, Allen PB, Greengard P, Luine V, McEwen BS (2004) Estrogen alters hippocampal dendritic spine shape and enhances synaptic protein immunoreactivity and spatial memory in female mice. Proc Natl Acad Sci USA 101:2185-2190.

Luine VN, Jacome LF, Maclusky NJ (2003) Rapid enhancement of visual and place memory by estrogens in rats. Endocrinology 144:2836-2844.

Mumby DG (2001) Perspectives on object-recognition memory following hippocampal damage: lessons from studies in rats. Behav Brain Res 127:159-181.

Nicholas A, Munhoz CD, Ferguson D, Campbell L, Sapolsky R (2006) Enhancing cognition after stress with gene therapy. J Neurosci 26:11637-11643.

Oitzl MS, de Kloet ER (1992) Selective corticosteroid antagonists modulate specific aspects of spatial orientation learning. Behav Neurosci 106:62-71.

Oitzl MS, Reichardt HM, Joëls M, de Kloet ER (2001) Point mutation in the mouse glucocorticoid receptor preventing DNA binding impairs spatial memory. Proc Natl Acad Sci USA 98:12790-12795.

Okuda S, Roozendaal B, McGaugh JL (2004) Glucocorticoid effects on object recognition memory require training-associated emotional arousal. Proc Natl Acad Sci USA 101:853-858.

Pavlides C, Watanabe Y, McEwen B (1993) Effects of glucocorticoids on hippocampal long-term potentiation. Hippocampus 3:183-192.

Pavlides C, Kimura A, Magarinos AM, McEwen BS (1994) Type I adrenal steroid receptors prolong hippocampal long-term potentiation. NeuroReport 18:2673-2677.

Pavlides C, Kimura A, Magarinos AM, McEwen BS (1995) Hippocampal homosynaptic long-term depression/depotentiation induced by adrenal steroids. Neuroscience 68:379-385.

Roozendaal B (2003) Systems mediating acute glucocorticoid effects on memory consolidation and retrieval. Prog Neuropsychopharmacol Biol Psychiatry 27:1213-1223.

Rozeboom AM, Akil H, Seasholtz AF (2007) Mineralocorticoid receptor overexpression in forebrain decreases anxiety-like behavior and alters the stress response in mice. Proc Natl Acad Sci USA 11:4688-4693.

Stein-Behrens BA, Lin WJ, Sapolsky RM (1994) Physiological elevations of glucocorticoids potentiate glutamate accumulation in the hippocampus. J Neurochem 63:596-602.

Vazquez DM, Lopez JF, Morano MI, Kwak SP, Watson SJ, Akil H (1998) Alpha, beta, and gamma mineralocorticoid receptor messenger ribonucleic acid splice variants: differential expression and rapid regulation in the developing hippocampus. Endocrinology 139:3165-3177.

Wang H, Hu Y, Tsien JZ (2006) Molecular and systems mechanisms of memory consolidation and storage. Prog Neurobiol 79:123-135.

Yau JL, Noble J, Seckl JR (1999) Continuous blockade of brain mineralocorticoid receptors impairs spatial learning in rats. Neurosci Lett 277:4548 . 